\title{
review
}

\section{Surgical options in treating patients with primary hyperparathyroidism}

\author{
Masa Majcen ${ }^{1}$, Marko Hocevar² \\ ${ }^{1}$ General Hospital Celje, Celje, Slovenia \\ 2 Institute of Oncology Ljubljana, Ljubljana, Slovenia \\ Radiol Oncol 2020; 54(1): 22-32. \\ Received 5 November 2019 \\ Accepted 15 November 2019 \\ Correspondence to: Maša Majcen, M.D., General Hospital Celje, Oblakova 5, SI-3000 Celje, Slovenia. E-mail: masa.majcen@gmail.com \\ Disclosure: No potential conflicts of interest were disclosed.
}

Background. Primary hyperparathyroidism is the third most common endocrine disorder for which surgical procedure called parathyroidectomy is the most effective treatment. Since the early $20^{\text {th }}$ century, parathyroid surgery has improved extensively. With the advances in preoperative imaging and with understanding the causes of disease, new and minimally invasive surgical approaches overrode the standard bilateral exploratory operations. Directed parathyroidectomy is currently the standard technique for treatment of primary hyperparathyroidism worldwide.

Conclusions. Surgery is the only definitive treatment of primary hyperparathyroidism. The most appropriate type of surgical procedure depends on the number and localization of the hyperactive parathyroid glands, availability of modern imaging techniques, limitation of each type of procedure and expertise.

Key words: primary hyperparathyroidism; minimally invasive parathyroidectomy; directed parathyroidectomy; endoscopic parathyroidectomy; bilateral neck exploration

\section{Introduction}

Primary hyperparathyroidism (PHPT) is a disease of parathyroid glands that results from their overactivity. The estimated incidence of PHPT is approximately two cases per 1,000 persons per year. ${ }^{1}$ About $80 \%$ of patients have no symptoms; the disease is generally detected during random laboratory blood test. In countries where biochemical test are not commonly used, classical symptoms and signs tend to predominate. Surgical resection is the only potentially curative treatment and remains the leading treatment option for PHPT. If the patient does not meet surgical criteria or refuses surgery, specific pharmacological therapy or close monitoring is recommended. ${ }^{1}$

\section{The evolution of parathyroid surgery}

Parathyroid glands were first discovered in Indian rhinoceros by English biologist Sir Richard Owen in $1850 .{ }^{2,3}$ Only 30 years later, Ivar Sandstörm from Sweden was the first person to describe the location and blood flow of glands in humans and coined the term glandulae parathyroidiae. ${ }^{1}$

It has taken many years for scientists to understand the mechanism of action of parathyroid glands. With the rapid development of thyroid surgery (thyroidectomy) in the early $20^{\text {th }}$ century, common occurrences of tetany were observed. ${ }^{2}$ The fact that hypocalcaemia after parathyroid surgery is the definitive cause of tetany was not wholly ac- 
cepted for several decades. ${ }^{3}$ It was not until 1909 that the first evidence of the relationship between parathyroid glands and the metabolism of calcium was published by MacCallum and Voegtil. ${ }^{2-4}$

Parathyroid surgery as a possible treatment of parathyroid disease was first mentioned in $1915 .^{5}$ Through the years, several scientists insisted on the connection between parathyroid disease and bone disease. Schlagenhaufer suggested the removal of a solitary tumour of the parathyroid gland in attempt to cure bone disease (described as osteitis fibrosa cystica). ${ }^{2,3}$ The professional public was sceptical because of the lack of evidence on the link between bone disease and the tumour. However, it took another 10 years before relationship between parathyroid disease and bone involvement was accepted. The first (successful) parathyroidectomy was performed by Felix Mandl in Vienna in 1925.5,6 Prior to the surgery, he initially tried to cure his patient (who had cystic bone lesions) with animal parathyroid extracts and transplantation of four fresh parathyroid glands, taken from the street accident victim. After surgical excision of the tumour with preservation of the other three parathyroid glands, the patient's condition improved dramatically. The patient later died from kidney failure. ${ }^{2,5,6}$

After the first successful parathyroidectomy, the surgery became the main treatment of parathyroid disease. Like in any other surgery, however, there are potential risks and complications. In the early 1930s, many surgeons noted the occurrence of severe, life-threatening hypocalcaemia after surgery. 5,6 They (often unsuccessfully) tried to treat hypocalcaemia with a high-calcium and lowphosphate diet or intravenous calcium administration. ${ }^{7}$ Churchill and Cope's detailed description of parathyroid surgery facilitated the development of surgical treatment. They employed frozen sections and routinely performed biopsy from normal tissue to rule out hyperplasia. ${ }^{4}$ They also wrote recommendations for the course of surgery (based on their experience in 30 observed patients) according to the likelihood of parathyroid hyperplasia or multiple adenomas. With the development of histology and the understanding of function of parathyroid glands, their variations in the number and location, surgical success rate slowly became higher. ${ }^{7}$ Near the end of the $20^{\text {th }}$ century; high-tech diagnostic imaging techniques became powerful medical tools that allowed surgeons to operate with minimal invasion to the patient. Since then, the use of minimally invasive surgery has expanded widely and has almost completely replaced the conventional surgical techniques. ${ }^{5,6}$

\section{Anatomy of parathyroid glands}

The anatomy of parathyroid glands is highly variable. ${ }^{78} \mathrm{~A}$ fundamental understanding of both surgical anatomy and embryology of parathyroid glands is key to successful parathyroid surgery. Nowadays, precise preoperative localization facilitates successful surgical therapy, but a good knowledge of anatomy remains irreplaceable. ${ }^{7}$

Parathyroid glands are yellowish brown endocrine glands, measuring about $6 \times 4 \times 2 \mathrm{~mm}$ and weighing on average 20 to $40 \mathrm{mg} .{ }^{8,9}$ The majority of glands are oval, bean shaped or spherical and usually lie on the posterior aspect of the thyroid lateral lobes. ${ }^{9}$ Each gland is separated from the thyroid gland by a thin connective tissue capsule. Most people $(85 \%)$ have four parathyroid glands ${ }^{7-10}$ :

- Two superior glands, each of which embryologically arises from the fourth pharyngeal pouch. They lie $1-2 \mathrm{~cm}$ above the intersection of the inferior thyroid artery and the recurrent laryngeal nerve at the level of the inferior margin of the cricoid cartilage.

- Two inferior glands, each of which embryologically arises from the third pharyngeal pouch. They are usually (in $70-80 \%$ of people) located in the close proximity to the inferior margin of the thyroid gland.

A small number of patients have three or even more than four glands. ${ }^{8}$ Rarely, parathyroid glands will be located elsewhere in the neck or in the chest. They are so-called ectopic parathyroid glands. ${ }^{9}$ Ectopic superior parathyroid glands can be found para- or retropharyngeal, retrotracheal or in the upper mediastinum, rarely also within the thyroid capsule. ${ }^{7}$ The location of the lower parathyroid glands is more variable due to their longer embryologic migration pathway. They can be found anywhere along this embryologic tract: along the thyrothymic ligament, the carotid sheath, in the anterior mediastinum etc. ${ }^{7-9}$

The upper and lower parathyroid glands are supplied by branches of the inferior thyroid artery, which is the major blood supply for lower poles of thyroid. ${ }^{78}$ To preserve the vascular supply to the parathyroid glands, the ligation of branches of inferior thyroid artery in thyroidectomy should be done with caution as close as possible to the thyroid capsule. Venous drainage is carried out by the inferior, middle, and superior thyroid veins, which drain into the internal jugular vein. ${ }^{78}$ 
TABLE 1. Biological actions of parathyroid hormone (PTH) in the body. PTH increases the serum calcium concentration and lowers the serum phosphate concentration

\begin{tabular}{ll}
\hline Organ system & Function of PTH \\
\hline Kidneys (leading role) & $\begin{array}{l}\text { It increases calcium and decreases phosphate reabsorption, stimulates calcitriol production by increasing } \\
\text { the synthesis of the enzyme 1- } \alpha \text { hydroxylase in proximal tubules. } \\
\text { It raises calcium levels in blood by increasing bone destruction (via osteoblast-mediated activation of } \\
\text { Osteoclasts) and decreasing the formation of new bone. }\end{array}$ \\
Skeletal & $\begin{array}{l}\text { It increases calcium absorption by stimulating the production of 1,25-dihydroxycholecalciferol. } \\
\text { Other (minor role, experimental) }\end{array}$ \\
$\begin{array}{l}\text { Metabolic effects (reduced glucose tolerance, changes in fat metabolism), effects on the liver, adipose } \\
\text { tissue, cardiovascular system, neuromuscular function. }\end{array}$
\end{tabular}

TABLE 2. Clinical presentation of developed primary hyperparathyroidism (PHPT). Symptoms and clinical signs are associated with an elevated serum calcium concentration and/or increased secretion of parathyroid hormone (PTH)

\begin{tabular}{ll}
\hline Organ system & Symptoms and clinical signs \\
\hline General & anorexia, polyuria, polydipsia, weight gain, anaemia \\
Skeletal & osteitis fibrosa cystica (bone pain, decreased bone density or generalized osteoporosis, pathological fractures) \\
Kidney & kidney stones, renal parenchymal calcifications, nephrocalcinosis, chronic renal impairment \\
Neuromuscular & proximal muscle weakness, depression, decline in cognitive ability, psychosis \\
Cardiovascular & arterial hypertension, arrhythmias, left ventricular hypertrophy, vascular wall and myocardial calcification \\
Gastrointestinal & nausea, vomiting, constipation, ulcer disease, pancreatitis \\
Rheumatological & gout, pseudogout \\
\hline
\end{tabular}

\section{Hyperparathyroidism}

Hyperparathyroidism is a common endocrine disorder in which one or more parathyroid glands become overactive. ${ }^{1}$ They produce and secrete parathyroid hormone (PTH). PTH affects several organ systems through multiple mechanisms. Its main role is regulation of calcium homeostasis but is important as well in vitamin $\mathrm{D}$ and phosphate regulation (see Table 1). ${ }^{1,8,11-14}$ The most common cause of hyperparathyroidism is excessive and unregulated production of PTH by parathyroid glands, socalled PHPT. More than $80 \%$ of PHPT is caused by a single benign parathyroid neoplasm - adenoma. ${ }^{8}$ According to epidemiological research data, PHPT is the third most common endocrine disorder. ${ }^{11,12}$ It affects 2 in 1,000 people per year in western world and its incidence increases with age, with a peak incidence in the seventh decade of life. The risk of developing PHPT is 4-fold higher in women. ${ }^{1,8}$ On the other hand, secondary hyperparathyroidism refers to compensatory physiological response of parathyroid glands to chronic hypocalcaemic state due to other pathological processes (mostly kidney disease). ${ }^{1,8,12}$ Unlike PHPT, a disease outside of the parathyroids causes all of the parathyroid glands to become enlarged and hyperactive. Patients with long-standing secondary hyperparathyroidism may develop autonomous parathyroid function due to random mutation in one of the parathyroid glands called tertiary hyperparathyroidism. ${ }^{12}$ The clinical picture is usually similar and difficult to distinguish from PHPT. The following paper focuses on the diagnosis and treatment of PHPT.

\section{Clinical presentation and diagnostics}

Nowadays, hyperparathyroidism is usually found accidentally during routine biochemical blood tests. ${ }^{15}$ Patient history and clinical presentation are often not helpful. ${ }^{16}$ Hyperparathyroidism should be considered in asymptomatic patients with elevated serum calcium levels. An atypical form of PHPT with normocalcemia and elevated PTH levels is rare (some authors argue that an atypical PHPT is an early form of the PHPT)..$^{1,7,15}$ Other possible causes of hypercalcaemia should always be ruled out. ${ }^{15}$ Occasionally, non-specific symptoms are found in presumed asymptomatic patients with a more detailed questioning (for example fatigue, depression, neuromuscular symptoms). In the past, the disease was diagnosed in the more advanced phase 
TABLE 3. The 2014 Fourth International Guidelines for the Management of Asymptomatic PHPT. Patients need to meet at least one of the following criteria to be advised to have surgery

\begin{tabular}{ll}
\hline Measurement & Criteria \\
\hline Age of patient & $<50$ years \\
Serum calcium concentration (above the upper reference value) & $>0.25 \mathrm{mmol} / \mathrm{L}(1.0 \mathrm{mg} / \mathrm{dL})$ \\
Skeletal injury & bone mineral density (DXA): T-sCore $<-2.5$ SD* \\
& spinal fracture (proven by XR, CT, MRI or VFA) \\
& creatinine clearance $<60 \mathrm{~mL} / \mathrm{min}$ \\
Renal impairment & kidney stones or nephrocalcinosis (proven by XR, US or CT) \\
& 24 -hour calcium in urine $>10 \mathrm{mmol} / \mathrm{L}$ ( $400 \mathrm{mg} / \mathrm{day})$ or increased risk for \\
\hline
\end{tabular}

* measured on the lumbar spine, hip, femoral neck or distal third of the radius

$\mathrm{CT}$ = computed tomography; DXA = dual-energy $\mathrm{x}$-ray absorptiometry; MRI = magnetic resonance imaging; $\mathrm{SD}=$ standard deviation; US = ultrasound; VFA = vertebral fracture assessment; $X R=x$-ray imaging

or so-called classical form, which is less commonly seen today.$^{16}$ The developed clinical picture is very diverse due to the broad action of PTH and different calcium concentrations (Table 2). , $12,14-16^{-16}$

Laboratory measurement of serum calcium concentrations should be obtained on at least two separate occasions. Hypercalcemia with concomitant elevated PTH levels confirms the diagnosis of PHPT.,15 Some patients have atypical form of hyperparathyroidism with elevated PHT levels in the absence of hypercalcemia. ${ }^{16}$ This phenotype of PHPT is called normocalcemia with abnormally high PTH. Different secondary causes need to be excluded before diagnosis of atypical PTH is made. ${ }^{15}$ At least the serum concentration of phosphate, chlorine, alkaline phosphatase, vitamin $\mathrm{D}$ and the patient's acid-base balance must be checked, as well as basic examination of urine and urinary sediment. . $^{1,15,16}$

All patients with diagnosis of PHPT (including asymptomatic patients) should undergo a urinary tract ultrasound..$^{15}$ Other diagnostic procedures (dual-energy x-ray absorptiometry, bone x-ray, bone biopsy) should be performed only if there is reasonable clinical suspicion. ${ }^{1,15}$

After the diagnosis of PHPT is established, localization studies need to be done to determine if minimally invasive surgical treatment can be performed. ${ }^{13}$ The localization studies should not be used to diagnose the disease. Parathyroid glands can be imaged with multiple modalities. The neck ultrasound, $\mathrm{CT}$, scintigraphy or newer hybrid imaging PET/CT and SPECT/CT can be used. ${ }^{17-20}$ ${ }^{99 m}$ Tc-sestamibi scan (complemented with neck ultrasound) is still the most commonly used method in many countries. ${ }^{18}$ Sestamibi is a small protein la- belled with the radio-pharmaceutical technetium$99 \mathrm{~m}$. The scan is performed by injecting small amount of radioactive material, which is then absorbed by overactive parathyroid gland. The gamma camera detects radioactive material and shows position of parathyroid glands. Different studies reported the sensitivity of ${ }^{99 \mathrm{~m}} \mathrm{Tc}$-sestamibi between $77 \%$ and $89 \%$, but up to one-third of patients with adenomas could be sestamibi negative. ${ }^{18}$

$\mathrm{PET} / \mathrm{CT}$ is a newer and promising tool for localization of parathyroid adenomas. Different PET tracers can be used $\left({ }^{18} \mathrm{~F}-\right.$-fluorodeoxyglucose, ${ }^{18} \mathrm{~F}$-fluorocholine (FCH) etc.). ${ }^{19,21}$ Many studies are comparing FCH PET/CT with conventional imaging modalities. Behera and Damle reported the incremental role of PET/CT over sestamibi scan in 2016 because of better spacial resolution, possible detection of smaller adenomas and reduced scanning time. ${ }^{18,20}$ Retrospective study by Hočevar et al. analysed the results of preoperative localization with FCH PET/CT in 151 patients with PHPT. ${ }^{21}$ Choline is a precursor molecule for a major component of the cell membrane and is taken up by hyperfunctioning parathyroid cells and neoplastic cells. They concluded that FCH PET/CT is a reliable preoperative localization test prior to directed parathyroidectomy, with operative confirmation of location and therapy success rate above $95 \% .{ }^{21}$

\section{Indications and contraindications for surgical treatment}

The surgery is indicated in all patients with clinical symptoms of PHPT who agree to be treated with 


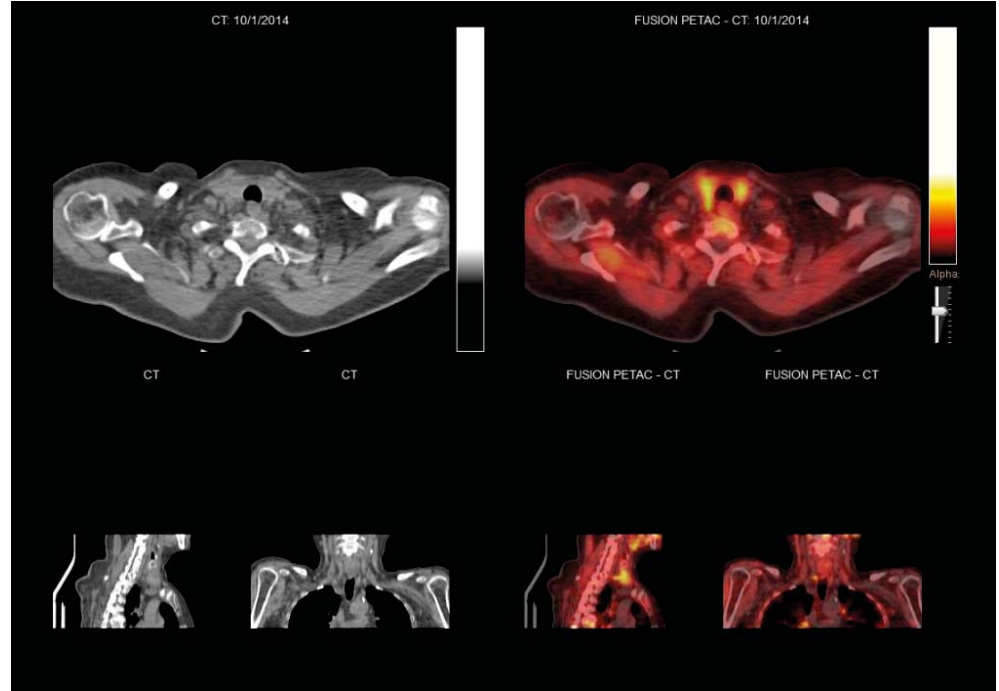

FIGURE 1. ${ }^{18} \mathrm{~F}$-fluorocholine (FCH) PET-CT fusion images of patient with pathologic uptake in the right lower parathyroid gland (solitary adenoma).

surgery. ${ }^{22}$ Successful surgical intervention can completely cure the disease. ${ }^{15}$ On the other hand, not all authors agree that surgery is beneficial for asymptomatic patients with PHPT. Differences in opinion arise mainly due to poor knowledge of the natural course of the disease, benefits of the surgical procedure and appropriate timing of surgery. ${ }^{1,16,22}$ The indications for surgery in asymptomatic patient with PHPT according to the Fourth International Guidelines for the Management of Asymptomatic PHPT are presented in Table $3 .^{23}$

If the patient is a suitable candidate for surgery according to the guidelines, possible contraindications for surgery must be considered. ${ }^{23,24}$ Surgery is absolutely contraindicated in patients with familial hypocalciuric hypercalcemia $(\mathrm{FHH})$, because it does not result in cure. ${ }^{25} \mathrm{FHH}$ is an autosomal dominant disorder that is often confused with PHPT due to similar laboratory findings (normoor hypercalcemia with elevated PTH). The major feature that distinguishes FHH from PHPT is 24-hour urinary calcium excretion. Urinary calcium concentration below $100 \mathrm{mg}$ in 24-hour urine is diagnostic for FHH. ${ }^{12,24.25}$ The relative contraindications are prior vocal cord injury, contralateral laryngeal nerve injury and symptomatic cervical disc herniation (parathyroid surgery is performed in neck hyperextension, which may worsen the condition). ${ }^{25}$ The contraindications listed above are common and apply to all types of surgical procedures on the parathyroid glands. Each surgical approach also has specific contraindications, which are mentioned in the descriptions of individual approaches.

\section{Preoperative management of patients}

Once the diagnosis of PHPT has been confirmed, determining the best approach for surgery depends on several factors. Before making any decision, the surgeon checks and evaluates the patient's medical history, family medical history, patient's regular treatment, the most recent laboratory results, etc. ${ }^{24,25}$ Specific tests (if not already performed) based on clinical symptoms may need to be done to check for the involvement of individual organs (kidney, bone, etc.). The preoperative examination should include at least the following ${ }^{1,24,25}$ :

- serum calcium levels and intact parathyroid hormone (iPTH);

- serum value of 25-hydroxy vitamin D;

- serum creatinine concentration;

- 24-hour urinary calcium concentration and creatinine values and

- serum thyroid-stimulating hormone (TSH) and thyroxine levels.

Accurate preoperative localization is crucial for successful surgical outcomes (particularly for minimally invasive parathyroidectomy). It helps to determine the location and number of hyperfunctioning parathyroid glands and it is very important in the case of recurrent neck procedures. ${ }^{18}$ In some western Europe countries conventional imaging with ${ }^{99 \mathrm{~m} T c-s e s t a m i b i}$ was successfully replaced by neck ultrasound and FCH PET-CT imaging (Figure 1). ${ }^{21}$ In some cases, additional imaging (e.g. CT, MRI) is required. Invasive procedures (selective venous sampling, selective arteriography) are reserved for patients who have had prior neck surgery and require reoperative surgery ${ }^{18,23}$ Due to the frequent concomitant thyroid and parathyroid disease, preventive preoperative analysis of the thyroid gland is performed to avoid increased complications from reoperations. ${ }^{26}$

\section{Types of surgical treatment of primary hyperparathyroidism}

The surgeon decides on the type of surgical procedure based on the patient's anamnesis, clinical status and results of preoperative examinations. ${ }^{24}$ The patient should be informed of the procedure that is indicated, possible surgical complications 
and the overall course of treatment. ${ }^{25}$ Today, there are several different surgical approaches that are further described below. With the development of medicine, classical surgical procedures are increasingly being replaced by new approaches that seek to minimize interventions into the human body. ${ }^{7}$ There are many different tools/procedures available today to perform surgery as accurately as possible, reduce the number of surgical complications and surgery repetitions, e.g. intraoperative neuromonitoring for identification of the recurrent laryngeal nerve, intraoperative iPTH assay, intraoperative frozen section when malignant tissue is suspected etc. ${ }^{7,24,25,27}$

\section{Bilateral neck exploration}

Bilateral neck exploration (also standard, open, conventional parathyroidectomy) is a traditional surgical approach for the treatment of PHPT. Due to the development of less invasive procedures, its application is decreasing. ${ }^{7}$ During the operation, the surgeon exposes all four parathyroid glands, therefore precise preoperative localization is not required (but it may be helpful). Parathyroid glands identification can be challenging even for a skilled surgeon due to their unpredictable location. ${ }^{22,25}$ According to literature data the success rate of surgery is $95 \%$ in experienced hands (the success of the procedure depends directly on the experience of the surgeon) ${ }^{8,25}$ In the past, a biopsy of all four parathyroid glands was used to histologically prove pathological parathyroid tissue. ${ }^{28}$ Due to the high risk of bleeding and postoperative hypoparathyroidism, it is no longer recommended. ${ }^{25,28}$

Current indications for bilateral parathyroidectomy include $25,28-30$ :

- unreliable or inaccessible preoperative imaging;

- preoperative imaging is contraindicated (pregnancy etc.);

- multiple parathyroid lesions;

- ectopic parathyroid glands location (inaccessibility with minimally invasive intervention),

- familial PHPT;

- concurrent thyroid surgery and

- other contraindications for minimally invasive intervention (measurement of iPTH is not available etc.).

The surgical approach has not changed significantly since the first bilateral parathyroidectomy in 1925.7 Surgery is usually performed under general anaesthesia, local anaesthesia is rarely used (depending on the experience of the surgical and anaesthesia team). ${ }^{30.31}$ The patient is lying on the operating room table in a supine position with his upper body at 30 degrees. ${ }^{25}$ The surgeon makes a 3 to $5 \mathrm{~cm}$ transverse incision (initially the incision was longer) about $2 \mathrm{~cm}$ above the collarbone. Subcutaneous fat and platysma are divided and the infrahyoid strap muscles are retracted for optimal exposure of thyroid. Using upward and medial digital retraction of the thyroid gland, the surgeon enters the "parathyroid" space, ${ }^{7,25}$ Once the important structures come into view (inferior thyroid artery and recurrent laryngeal nerve), the surgeon continues with the identification of the parathyroid glands. ${ }^{31}$ Caution should be used when removing the parathyroid tissue and during connective tissue manipulation, because the rupture of the parathyroid capsule can result in parathyromatosis. ${ }^{25}$ The surgeon must identify all four parathyroid glands but remove only the abnormal ones. ${ }^{31}$ When in any doubt, frozen section can be done to confirm that the tissue excised is parathyroid in origin. In the event of accidental removal of healthy parathyroid tissue, reimplantation of the tissue should be considered (usually into the brachioradial forearm muscle). ${ }^{7,25}$ After successful removal of pathological glands and proper haemostasis, the surgeon approximates the retracted infrahyoid strap muscles and closes the wound with a continuous intradermal suture. ${ }^{25,28-31}$

The most common complication of bilateral neck exploration is recurrent or persistent hyperparathyroidism that results from missed adenoma or parathyromatosis. Rarely, the cause of recurrent hyperparathyroidism is new neoplasia (most commonly in previously unrecognized familial PHPT or multiglandular disease, for example multiple endocrine neoplasia [MEN syndrome]). ${ }^{25}$ As well as the success of the operation, the rate of complication depends directly on the experience of the surgeon (to be considered experienced, surgeon should perform at least 50 procedures per year). ${ }^{25}$ During the procedure, the superior or recurrent laryngeal nerves on either side can be damaged. ${ }^{8}$ Damage can cause long-term hoarseness or voice loss. According to the literature, permanent nerve injury occurs in less than $1 \%$ of patients. ${ }^{8,33}$ Transient nerve injuries (neuropraxia) are more common and have different recovery times, complete recovery generally occurs within the first six months. Postoperative hypocalcaemia is usually transient and occurs in $25 \%$ of patients. ${ }^{25,33}$ It is most often due to slow reactivation of healthy parathyroid glands, which have been suppressed 


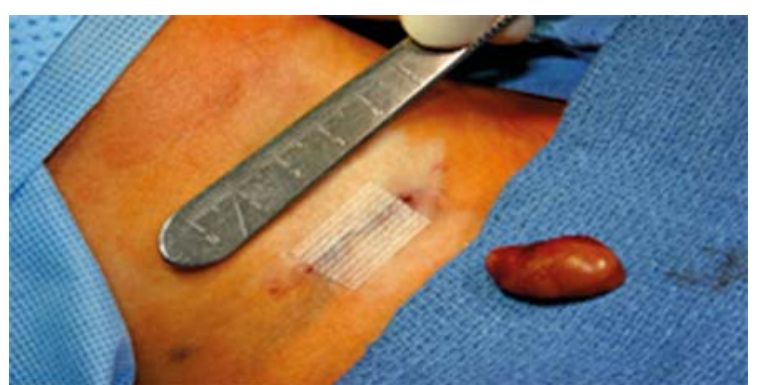

FIGURE 2. Directed parathyroidectomy. The image shows the incision site and the removed parathyroid tissue.

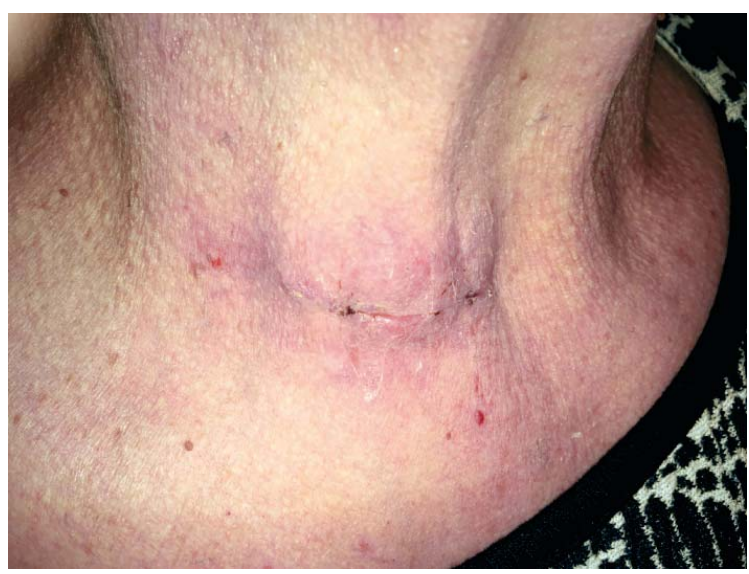

FIGURE 3. Appearance of the incision and surrounding skin 10 days after performing directed parathyroidectomy.

by overactive pathological parathyroid glands. After removal of the adenoma, the remaining suppressed glands will eventually regain their functional capacity. $8,25,32$ Patients with low blood calcium levels complain of numbness and tingling in their fingertips and toes, very low concentrations can result in involuntary movements and severe muscle spasms. ${ }^{25,33}$ Postoperative bleeding is a rare but dangerous complication, which may lead to respiratory problems and wound infection. ${ }^{25,33}$

\section{Minimally invasive parathyroidectomy}

The term minimally invasive parathyroidectomy (also focal, selective parathyroidectomy) has been used since the 1990s to describe newer types of surgeries on parathyroid glands. ${ }^{34,35}$ There is no exact definition of the term, but some experts define it as a procedure with minimal dissection (excision of the adenoma without the removal of non-pathological parathyroid glands) and a wound smaller than $2.5 \mathrm{~cm} .^{7}$ Worldwide, the term currently includes the following procedures: directed parathyroidec- tomy, endoscopic parathyroidectomy (total endoscopic, video-assisted and robotic parathyroidectomy) and isotope-guided parathyroidectomy. ${ }^{34} \mathrm{In}$ 2009, the European Society of Endocrine Surgeons (ESES) confirmed that in keeping with prescribed indications and contraindications, minimally invasive parathyroidectomy is a safe and reliable procedure. $^{35}$

\section{Directed parathyroidectomy}

With the development and improvement of preoperative imaging diagnostics, parathyroid surgery is focused on minimally invasive surgery. 5,6 Accurate preoperative localization (with neck ultrasound, sestamibi scan and/or FCH PET-CT) is critical to the success of this type of surgery. ${ }^{17-20}$ Directed parathyroidectomy is a procedure recommended for patients with solitary adenoma. ${ }^{7}$ Worldwide, this type of surgery accounts for $70 \%$ of all minimally invasive procedures. ${ }^{34}$ The operation is usually performed under general anaesthesia but can be also performed under regional anaesthesia (usually deep cervical block). Regional anaesthesia prevents side effects of general anaesthesia and at the same time allows for intraoperative testing of superior and recurrent laryngeal nerve (by evaluating the patient's speech). ${ }^{36}$ Depending on the location of the adenoma, a central or lateral 2 to $4 \mathrm{~cm}$ long incision is made at the neck (see Figure 2).,13 The surgeon identifies and removes only the pathological parathyroid gland (or solitary adenoma). ${ }^{25}$ Other parathyroid glands are not exposed. Since preoperative imaging is not completely reliable, majority of surgeons use iPTH to determine the surgery's success. ${ }^{13} \mathrm{iPTH}$ levels are determined at anaesthesia induction (general or regional) and 5-10 min after adenoma removal. According to Miami criteria, successful parathyroidectomy was defined as a $>50 \%$ decrease of iPTH level compared to the level measured before removal. The half-life of iPTH in plasma is $3-5$ min. ${ }^{37,38}$ Directed approach reduces the invasiveness of the surgery and shortens the surgery and hospitalization time. ${ }^{13,32,35}$ Published research also demonstrates a lower risk of operative complications (postoperative hypocalcaemia, recurrent laryngeal nerve damage) and death, compared to bilateral neck exploration., 73,25 The patient recovers faster, with less pain and a better aesthetic result (smaller postoperative scar) (Figure 3).25,32 The procedure is not appropriate for patients with multiglandular disease, thyroid disease that also requires surgery or a family history of MEN syndrome. ${ }^{13,25,27,28}$ 
In the last five years, FCH PET-CT has proven to be the most sensitive and specific method in preoperative localization of pathologically changed parathyroid glands. ${ }^{39}$ Its reliability to exclude multiglandular disease is so high that iPTH monitoring can be safely abandoned. ${ }^{40}$ This shortens the surgery time by 30 minutes, which is very significant given that the average duration of directed parathyroidectomy is less than 20 minutes.

According to a 2007 analysis by Westerdahl et al., the long-term results of directed parathyroidectomy with iPTH monitoring are comparable to bilateral neck exploration. ${ }^{41}$ Due to the benefits of this procedure primarily in the early postoperative period and the comparable risk of PHPT recurrence, this procedure is (with the patient's consent and the absence of contraindications) indicated as the procedure of choice for removing preoperatively proven solitary adenoma. ${ }^{41}$

\section{Endoscopic parathyroidectomy}

Endoscopic parathyroidectomy is a surgical technique using an endoscope. ${ }^{7}$ It is divided into two types, depending on the course: total endoscopic and video-assisted parathyroidectomy, which is a combination of endoscopic intervention and open surgical approach. ${ }^{34}$ In addition to a directed approach, the endoscope also allows us to perform the entire procedure through small incision wound and with small tissue damage. PHPT is a disease that is otherwise an "ideal disease" for endoscopic surgery, since ${ }^{34,42-44}$ :

- in most cases, the cause is a benign tumour,

- the tumour is mostly smaller than $3 \mathrm{~cm}$ and

- there is no need for surgical reconstruction after removal of small amount of tissue.

Endoscopic surgery has similar advantages to bilateral parathyroidectomy as other minimally invasive procedures. Compared to directed parathyroidectomy, it is less invasive, it results in less postoperative pain and a better aesthetic result, but surgery is longer, technically more demanding, and requires more expensive surgical equipment. ${ }^{45}$ General anaesthesia is always required. ${ }^{34}$ Endoscopic intervention is indicated in patients with sporadic PHPT and solitary adenoma, which should be confirmed by preoperative imaging. ${ }^{43}$ The procedure is not appropriate in the case of intrathyroid parathyroid adenoma, multiple parathyroid involvement, previous neck surgery, suspected parathyroid cancer, familial PHPT, secondary and tertiary hyperparathyroidism, goitre and in the obese patients. ${ }^{25,34,43,45}$

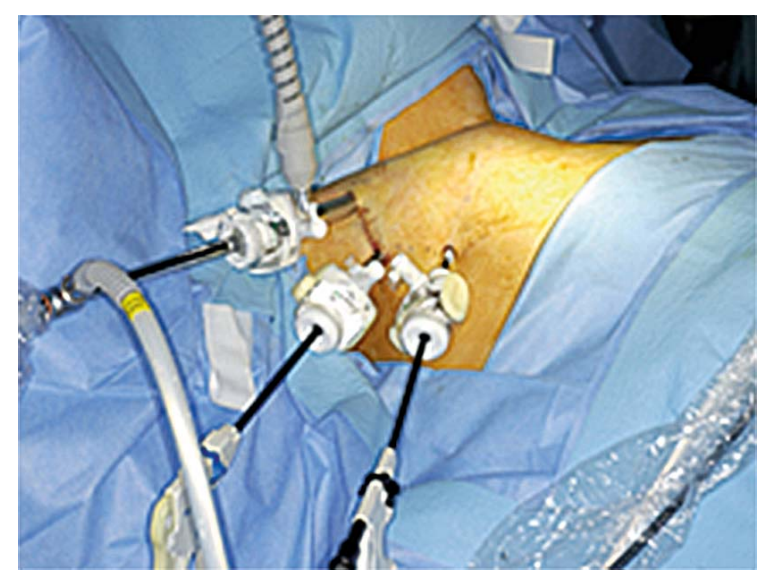

FIGURE 4. Total endoscopic parathyroidectomy.

\section{Total endoscopic parathyroidectomy}

Total endoscopic parathyroidectomy was first mentioned by Gagner et al. in 1996. ${ }^{46}$ Initial technique was carried out entirely under a steady gas flow, introduced through a central trocar. Nowadays, due to the limited exposure of structures, the central approach is used only for the more anteriorly located adenomas. ${ }^{46} \mathrm{~A}$ more frequently used technique is the lateral approach. The intervention begins with the insertion of three working channels for endoscopic instruments (sizes 2, 3 and $10 \mathrm{~mm}$ ) along the upper anterior margin of the sternocleidomastoid muscle (Figure 4). Carbon dioxide is insufflated to help the surgeon expose the area of work and show up the main structures (lateral border of the thyroid gland, recurrent laryngeal nerve, and both ipsilateral parathyroid glands) with lateral displacement of the infrahyoid muscles and remove the adenoma through a $10 \mathrm{~mm}$ trocar. ${ }^{47}$

According to the data, the conversion from endoscopic to traditional 'open' surgery is between $13.4 \%$ and $28 \% .{ }^{46,47}$ The causes are different, most often due to difficult dissection, bleeding or persistence of elevated iPTH after removal of the suspected solitary adenoma. ${ }^{25,46,47}$ The success of the endoscopic approach is very favourable in the short term (with the exception of a large number of conversion to the open mode), but the results of long-term studies are not yet available (intermediate results are encouraging) ${ }^{46,47}$ Fouyuet et al. operated on 200 patients with PHPT, 28\% were converted to open parathyroidectomy. After an average 13 months after endoscopic surgery, 197 patients $(98 \%)$ were reported to be cured. ${ }^{47}$ In another prospective study Vidal-Perez et al. performed 28 endoscopic lateral parathyroidectomies with no in- 
traoperative complications. They reported favourable outcome in 27 of the 28 patients $(96 \%)$ after an average of 22 months after procedure. ${ }^{48}$ The major limitation of the approach represents simultaneous bilateral adenomas, since the approach only allows simultaneous removal of the ipsilateral adenomas.

\section{Video-assisted parathyroidectomy}

Video-assisted parathyroidectomy is a combination of endoscopic and open surgery. It was first described in 1999 by Miccoli et al. ${ }^{35,49}$ The endoscope, with a smaller incision than used in other open procedures, allows the operator to visualize the operative field equally or better. This results in a smaller postoperative scar. The use of two to three times magnification of the operative field makes identification of anatomical structures easier and the risk of damage to the laryngeal recurrent nerve and other important structures is lower. The advantage is seen especially in the case of very posteriorly located parathyroid glands. ${ }^{25,34,35,50}$ According to data from Barczynski et al. from 2006, patients have less pain after this procedure compared with directed parathyroidectomy. There is reduced need for postoperative analgesia, a shorter incision wound and better cosmetic results, but at the cost of a longer intervention duration and higher intervention costs. The number of video-assisted parathyroidectomy conversions to the open-surgery and the length of hospitalization (compared to directed parathyroidectomy) were not statistically significantly different. There are no data available on long-term outcomes and effectiveness of the intervention. ${ }^{50}$

Video-assisted parathyroidectomy can be performed under local or general anaesthesia. ${ }^{49,50}$ The procedure is performed via a central incision located centrally above the collarbone. The endoscope of $5 \mathrm{~mm}$ in size is introduced (without a trocar) at an angle of 30 degrees via a lateral incision on the side of the pathological parathyroid gland..$^{35}$

Gas insufflation is not used in comparison with total endoscopic surgery. ${ }^{50}$ The endoscope is more mobile, but it requires an additional assistant to manage it. The procedure is performed in the same way as directed parathyroidectomy. ${ }^{35,50}$ Some authors also use endoscopes for bilateral exploratory parathyroidectomy in patients with multiglandular disease. . $5,31,35^{2}$

\section{Radioguided parathyroidectomy}

Radioguided parathyroidectomy is a type of minimally invasive surgery, during which the gamma probe is used to guide the surgeon towards the location of the pathological parathyroid gland. ${ }^{35}$ As with all minimally invasive procedures, good preoperative imaging plays an important role. ${ }^{17-20}$ Good interaction between the entire surgical team and nuclear medicine experts is a prerequisite for this type of surgical intervention. The patient is given an intravenous dose of ${ }^{99 \mathrm{~m}} \mathrm{Tc}$-sestamibi approximately 2 to 4 hours before the surgery. ${ }^{35}$ The uptake of sestamibi into the parathyroid adenoma cells depends on their activity. ${ }^{20}$ Intraoperatively, a gamma probe is then used to trace the location with the highest radioactivity (which must be at least 20\% higher than background neck activity). The surgeon thus determines the best incision site. ${ }^{35}$ However, the use of radioactivity to determine the location has its limitations. The thyroid pathology, which can have increased sestamibi uptake, may be mistaken for an abnormal parathyroid gland. When searching for radioactive ectopic parathyroids in the chest, high concentration of the isotope in the myocardium can be mistakenly detected. If the tissue resected is radioactive and the neck shows equal radioactivity after excision, the patient can be assumed cured. ${ }^{51}$ Nonetheless, iPTH assay is used to determine the complete excision of all hyperfunctioning tissue. ${ }^{21,51}$

The method described above is used only by few experienced surgeons. ${ }^{35}$ It is mostly used as an alternative method or just as an aid for the surgery. According to Burkey et al., the reliability of the gamma probe is only $66 \%$ and therefore is not a consistently reliable tool for routine use. ${ }^{51}$

\section{Robotic parathyroidectomy}

Robotic-assisted parathyroidectomy is a modern technique and it represents the new generation in the evolution of minimally invasive parathyroidectomy techniques..$^{52}$ The beginnings of telerobotic surgical technology go back to the early $21^{\text {st }}$ century when a new established idea of robotic-assisted surgery offered solutions in overcoming the limitations associated with endoscopic techniques (twodimensional view of the operative filed, limited manipulation with surgical instruments, need for carbon dioxide insufflation and assistants during surgery). ${ }^{52,53}$ In 2011, Tolley et al. introduced and performed the first robotic-assisted parathyroidec- 
tomy without the scar in the neck (by infraclavicular incision), using the da Vinci ${ }^{\oplus}$ surgical system..$^{53}$

As for every other minimal invasive surgical approach, preoperative imaging studies that assist in the localization of lesions have been key elements in patients' selection for targeted robotic-assisted parathyroid surgeries. ${ }^{17-20,54}$ Before robotic-assisted parathyroidectomy is preformed, parathyroid adenoma should be properly localized..$^{54}$ Tolley et al. preformed first robotic-assisted surgery (RAS) for parathyroidectomy in which they made an incision in infraclavicular region on the side of the lesion. Following that, three other small incisions had been performed in the axillary line on the ipsilateral side without using insufflation. Adenoma was successfully removed in all 11 patients that were represented in this pilot study for evaluating robotic parathyroidectomy; although it should be noted that during one of the RAS procedures the surgeon had to switch to the conventional open approach due to unsuitable habitus of the patient. Nevertheless, the robotic procedure took an average of 61 minutes. ${ }^{53}$ Many improvements and upgrades have since been introduced into this minimal invasive surgical technique. Nowadays, only one small incision is necessary for RAS. ${ }^{2}$ However, due to high prices of robotic systems and other equipment, robotic-assisted surgical technique in parathyroidectomy has been an alternative procedure only in selected medical centres in the USA and Great Britain. ${ }^{53,54}$ There are only few known studies about RAS in parathyroidectomy, yet all the known data supports robotic-assisted parathyroidectomy as an equally successful and safe approach for treatment of PHPT as conventional open or endoscopic assisted parathyroidectomy (level 2 and 3 diagnostic evidence).52 Despite an excellent cosmetic outcome due to small incision and a safe approach to the surgery, high expenses, limited equipment, long duration of the procedure and technical difficulties represent limitations in choosing ideal candidates for RAS. ${ }^{52-54}$

\section{Conclusions}

Parathyroid surgery is the only potentially curative treatment of PHPT. Over the last two decades, technological advances and better understanding of the disease have refined surgical techniques. Surgery represents the last step in the overall patient care. Disease recognition, a proper diagnostic process and preoperative preparation of the patient are crucial for choosing the best surgical pro- cedure. The aim of this paper was to discuss newer surgical techniques and their advantages and limitations in comparison to classical methods.

\section{References}

1. Madkhali T, Alhefdhi A, Chen H, Elfenbein D. Primary hyperparathyroidism. Ulus Cerrahi Derg 2016; 32: 58-66. doi: 10.5152/UCD.2015.3032

2. Rowlands BC. Hyperparathyroidism: an early historical survey. Ann R Coll Surg Engl 1972; 51: 81-90. PMID: 5077791

3. Coffey RJ. Historical highlights of hyperparathyroidism. Am Surg 1972; 38 649-52. PMID: 4566475

4. Churchill ED, Cope O. The surgical treatment of hyperparathyroidism based on 30 cases confirmed by operation. Ann Surg 1936; 104: 9-35. doi: 10.1097/00000658-193607000-00002

5. Dorairajan N, Pradeep PV. Vignette hyperparathyroidism: a glimpse into its history. Int Surg 2014; 99: 528-33. doi: 10.9738/INTSURG-D-13-00225.1

6. Sethi N, England RJA. Parathyroid surgery: from inception to the modern day. Br J Hosp Med (Lond) 2017; 78: 333-7. doi: 10.12968/hmed.2017.78.6.333

7. Lew II, Solorzano CC. Surgical management of primary hyperparathyroidism: state of the art. Surg Clin North Am 2009; 89: 1205-25. doi: 10.1016/j.suc.2009.06.014

8. Eržen J. [Parathyroid gland]. [Slovenian]. In: Smrkolj V, Pivec G, Turčić J, editors. Kirurgija. Celje: Grafika Gracer; 2014. p. 647-80.

9. Lyden LM, Wang TS, Sosa JA. Surgical anatomy of the parathyroid glands [internet]. UpToDate. [cited 2019 Oct 15]. Available at: https://www.uptodate. com/contents/surgical-anatomy-of-the-parathyroid-glands\#H13

10. Kochhar A. Parathyroid gland anatomy [internet]. Medscape. [cited 2019 Oct 15]. Available from: https://emedicine.medscape.com/article/1949105overview

11. Clarke BL. Epidemiology of primary hyperparathyroidism. J Clin Densitorm 2013; 16: 8-13. doi: 10.1016/j.jocd.2012.11.009

12. Fraser WD. Hyperparathyroidism. Lancet 2009; 374 (9684): 145-58. doi: 10.1016/S0140-6736(09)60507-9

13. El-Hady HA, Radwan HS. Focused parathyroidectomy for single parathyroid adenoma: a clinical account of 20 patients. Electron Physician 2018; 10: 6974-80. doi: 10.19082/6974.

14. Fuleihan GE, Brown EM. Parathyroid hormones secretion and action [internet]. UpToDate. [cited 2019 Oct 15]. Available at: https://www.uptodate. com/contents/parathyroid-hormone-secretion-and-action\#H22

15. Fuleihan GE, Silverberg SJ. Primary hyperparathyroidism: clinical manifestation [internet]. UpToDate; [cited 2019 Oct 15]. Available at: https://www. uptodate.com/contents/primary-hyperparathyroidism-clinical-manifestatio ns?topicRef=2029\&source=see_link\#H6

16. Bilezikian JP, Cusano NE, Khan AA, Liu JM, Marcocci C, Bandeira F. Primary hyperparathyroidism. Nat Rev Dis Primers 2016; 2: 16033. doi: 10.1038/ nrdp.2016.33

17. Hopkins CR, Reading CC. Thyroid and parathyroid imaging. Semin Ultrasound CT MR 1995; 16: 279-95. doi: 10.1016/0887-2171(95)90033-0

18. Prabhu M, Damle NA. Fluorocholine PET imaging of parathyroid disease. Indian J Endocrinol Metab 2018; 22: 535-41. doi: 10.4103/ijem. IJEM_707_17

19. Lundstroem AK, Trolle W, Soerensen CH, Myschetzky PS. Preoperative localization of hyperfunctioning parathyroid glands with 4D-CT. Eur Arch Otorhinolaryngol 2016; 273: 1253-9. doi: 10.1007/s00405-015-3509-9

20. Behera A, Damle NA. Incremental role of 18F-fluorocholine PET/CT over technetium-99m-labeled MIBI scan in hyperparathyroidism. Indian J Endocrinol Metab 2016; 20: 888-90. doi: 10.4103/2230-8210.192897

21. Hocevar M, Lezaic L, Rep S, Zaletel K, Kocjan T, Sever MJ, et al. Focused parathyroidectomy without intraoperative parathormone testing is safe after pre-operative localisation with 18F-Flouorocholine PET/CT. Eur J Surg Oncol 2017; 43: 133-7. doi: 10.1016/j.ejso.2016.09.016 
22. Nies C. [Diagnostics and treatment of primary hyperparathyroidism] [German]. Chirurg 2017; 88: 1069-80. doi: 10.1007/s00104-017-0517-x

23. Bilezikian JP, Brandi ML, Eastellet R, Silverberg SJ, Udelsman R, Marcocci C, et al. Guidelines for management of asymptomatic primary hyperparathyroidism: summary statement from the Fourth International Workshop. J Clin Endocrinol Metab 2014; 99: 3561-9. doi: 10.1210/jc.2014-1413

24. Fuleihan GE, Silverberg SJ. Primary hyperparathyroidism: diagnosis, differential diagnosis and evaluation. [internet]. UpToDate; c2019. [cited 2019 Aug 15]. Available at: https://www.uptodate.com/contents/primaryhyperparathyroidism-diagnosis-differential-diagnosis-and-evaluation?topic Ref=2031\&source=related_link

25. Perrier ND, Dickson PV, Figueroa AS. Parathyroid exploration for primary hyperparathyroidism [internet]. UpToDate; c2019. [cited 2019 Aug 15]. Available from: https://www.uptodate.com/contents/parathyroid-exploration-for-primary-hyperparathyroidism?search=focused $\% 20$ paratiroidectomy§ionRank=1\&usage type=default\&anchor=H132555 $5031 \&$ source=machineLearning \& selectedTitle $=81$ play18\#8is $1481 \mathrm{Hisp}$ lay850+\#1681\#1681\#1681\#1681\#1681\#1681\#1681\#1681+\#1681\#16 81\#1681\#1681\#1681\#1681\#1681\#1681\#1681+Hisplay850

26. Stang MT, Yim JH, Challinoret SM, Bahl S, Carty SE. Hyperthyroidism afte parathyroid exploration. Surgery 2005; 138: 1058-64. doi: 10.1016/j. surg.2005.09.011

27. Osamura RY, Hunt JL. Current practices in performing frozen sections for thyroid and parathyroid pathology. Virchows Arch 2008; 453: 433-40. doi: $10.1007 /$ s00428-008-0674-x

28. UW Health: Endocrine surgery. [internet]. Madison (WI): University of Wisconsin Hospitals and Clinics Authority; c2019. [cited 2019 Aug 15] Available at: https://www.uwhealth.org/endocrine-surgery/bilateral-exploration $/ 25440$

29. Oertlii D. [Indications for parathyroidectomy]. [German]. Praxis (Bern 1994) 2007; 96: 981-8. doi: 10.1024/1661-8157.96.24.981

30. Norman J. Parathyroid surgery: the standard technique [internet]. Montclair (NJ): EndocrineWeb; c2014. [cited 2019 Oct 15]. Available at: https://www. endocrineweb.com/conditions/hyperparathyroidism/parathyroid-surgerystandard-technique

31. AAES: How is parathyroid surgery performed? [internet]. [cited 2029 Oct 15]. USA: The American Association of Endocrine Surgeons; c2018. Available at: http://endocrinediseases.org/parathyroid/surgery_overview. shtml\#2

32. Bergenfelz A, Lindblom P, Tibblin S, Westerdahl J. Unilateral versus bilateral neck exploration for primary hyperparathyroidism: a prospective randomized controlled trial. Ann Surg 2002; 236: 543-51. doi 10.1097/00000658-200211000-00001

33. AAES: Parathyroid surgery: risks [internet]. USA: The American Association of Endocrine Surgeons; c2018. [cited 2019 Aug 15]. Available at: http:// endocrinediseases.org/parathyroid/surgery risks.shtml

34. Brunaud L, Li Z, Van Den Heede K, Cuny T, Van Slycke S. Endoscopic and robotic parathyroidectomy in patients with primary hyperparathyroidism. Gland Surg 2016; 5: 352-60. doi: 10.21037/gs.2016.01.06

35. Bellantone R, Raffaelli M, de Crea C, Traini E, Lombardi CP. Minimallyinvasive parathyroid surgery. Acta Otorhinolaryngol Ital 2011; 31: 207-15. PMID: 22065831

36. Pintaric TS, Hočevar M, Jereb S, Casati A, Novak Jankovic V. A prospective, randomized comparison between combined (deep and superficial) and superficial cervical plexus block with levobupivacaine for minimally invasive parathyroidectomy. Anesth Analg 2007; 105: 1160-3. doi: 10.1213/01. ane.0000280443.03867.12

37. Carneiro DM, Solorzano CC, Nader MC, Ramirez M, Irvin GL $3^{\text {rd }}$. Comparison of intraoperative iPTH assay (QPTH) criteria in guiding parathyroidectomy: which criterion is the most accurate? Surgery 2003; 134: 973-9. doi: 10.1016/j.surg.2003.06.001

38. Greif B, Hojski A, Crnjac A. Intraoperative parathyroid hormone assessment in patients with primary hyperparathyroidism - one-year experience. Acta Med Bio 2014; 7: 39-44.

39. Lezaič L, Rep S, Sever MJ, Kocjan T, Hočevar M, Fettich J. ${ }^{18} \mathrm{~F}$-flouorocholine $\mathrm{PET} / \mathrm{CT}$ for localization of parathyroid tissue hyperfunctioning in primary hyperparathyroidism: a pilot study. Eur J Nucl Med Mol Imaging 2014; 41: 2083-9. doi: 10.1007/s00259-014-2837-0
40. Hočevar M, Lezaič L, Rep S, Zaletel K, Kocjan T, Sever MJ, et al. Focused parathyroidectomy without intraoperative parathormone testing is safe after pre-operative localization with ${ }^{18} \mathrm{~F}$-fluorocholine PET/CT. Eur J Nucl Oncol 2017; 43: 133-7. doi: 10.1016/j.ejso.2016.09.016

41. Westerdahl J, Bergenfelz A. Unilateral versus bilateral neck exploration for primary hyperparathyroidism: five-year follow-up of a randomized controlled trial. Ann Surg 2007; 246: 976-80. doi: 10.1097/SLA.0b013e31815c3ffd

42. Cheng LHH, Ali E, Qureshi R. Indications and contraindications of minimally invasive video-assisted thyroidectomy and parathyroidectomy. [abstract]. BAOMS Annual Scientific Meeting, 22-22 June 2012, London, UK. Br J Oral Maxillofac Surg 2012; 50: S51-2.

43. Henry JF, Sebag F, Cherenko M, Ippolito G, Taieb D, Vaillant J. Endoscopic parathyroidectomy: why and when? World J Surg 2008; 32: 2509-15. doi: 10.1007/s00268-008-9709-3

44. Clerici T. Minimally invasive techniques in thyroid surgery. In: Oertli D, Udelsman R. Surgery of thyroid and parathyroid glands. Berlin: SpringerVerlag; 2012. p. 175-87.

45. Hessman O, Westerdahl J, Al-Suliman N, Christiansen P, Hellman P, Bergenfelz A. Randomized clinical trial comparing open with video-assisted minimally invasive parathyroid surgery for primary hyperparathyroidism. $\mathrm{Br}$ J Surg 2010; 97: 177-84. doi: 10.1002/bjs.6810

46. Henry JF, Sebag F, Tamagnini P, Forman C, Silaghi H. Endoscopic parathyroid surgery: results of 365 consecutive procedures. World J Surg 2004; 28 : 1219-23. doi: 10.1007/s00268-004-7601-3

47. Fouquet T, Germain A, Zarnegar R, Klein M, De Talance N, Claude Mayer J, et al. Totally endoscopic lateral parathyroidectomy: prospective evaluation of 200 patients. ESES 2010 Vienna presentation. Langenbecks Arch Surg 2010 395: 935-40. doi: 10.1007/s00423-010-0687-1

48. Vidal-Perez O, Valentini M, Baanante-Cerdena JC, Ginesta-Marti C, Fernandez-Cruz L, Garcia-Valdecasas JC. [Endoscopic lateral parathyroidectomy as surgical treatment for patients with primary hyperparathyroidism]. [Spanish]. Cir Cir 2016; 84: 15-20. doi: 10.1016/j.circir.2015.06.031

49. Miccoli P, Pichera A, Cecchini G, Conte M, Bendinelli C, Vignali E, et al. Minimally invasive, video assisted parathyroid surgery for primary hyperparathyroididm. J Endocrinol Invest 1997; 20: 429-30. doi: 10.1007/ BF03347996

50. Barczyński M, Cichoń S, Konturek A, Cichoń W. Minimally invasive video-assisted parathyroidectomy versus open minimally invasive parathyroidectomy for a solitary parathyroid adenoma: a prospective, randomized, blinded trial. World J Surg 2006; 30: 721-31. doi: 10.1007/s00268-005-0312-6

51. Burkey SH, Van Heerden JA, Farley DR, Thompson GB, Grant CS, Curlee KJ. Will directed parathyroidectomy utilize the gamma probe or intraoperative parathyroid hormone assay to replace bilateral cervical exploration as the preferred surgery for primary hyperparathyroidism? World J Surg 2002; 26: 914-20. doi: 10.1007/s00268-002-6618-8

52. Arora A, Garas G, Tolley N. Robotic parathyroid surgery: current perspectives and future considerations. ORL J Otorhinolaryngol Relat Spec 2018; 80: 195203. doi: $10.1159 / 000488355$

53. Tolley N, Arora A, Palazzo F, Garas G, Dhawan R, Cox J, et al. Robotic-assisted parathyroidectomy: a feasibility study. Otolaryngol Head Neck Surg 2011; 144: 859-66. doi: 10.1177/0194599811402152

54. Garas G, Arora A, Tolley N. Robotic surgery for the parathyroid glands. In: Grillone G, Jalisi S, editors. Robotic surgery of the head and neck. New York: Springer; 2015. p. 133-46. 